\title{
Progress on HERON: A Real-time Detector for P-P Solar Neutrinos
}

\author{
J.S. Adams, A. Fleischmann, Y.H. Huang, Y.H. Kim, \\ R.E. Lanou, H.J. Maris \& G.M. Seidel \\ Department of Physics, Brown University, Providence, RI 02912
}

\begin{abstract}
The HERON project is an R\&D effort to create a detector suitable for real-time, high rate measurement of neutrinos from both the p-p and ${ }^{7} \mathrm{Be}$ reactions in the Sun using superfluid helium as the target medium. Progress on studies of particle detection processes in superfluid, on development of sensors, on backgrounds, and on event energy and position measurement which are related to this goal are discussed.
\end{abstract}

\section{INTRODUCTION}

The study of solar neutrinos continues to play a major role in the effort to measure the properties of the neutrino families. The need to measure the total low energy neutrino flux and its composition is emerging as the next goal of solar neutrino research. The entire neutrino energy spectrum from all the reactions in the Sun extends from $0 \mathrm{MeV}$ up to nearly $19 \mathrm{MeV}$. Measuring as much of this spectrum as possible is a principal part of the program to establish whether or not neutrino oscillations are the cause of the observed discrepancies in fluxes measured at the Earth, and if that is the case, to measure as precisely as possible their mass and mixing parameters.

No single detection technique can suffice for all of the measurements needed to cover the total energy range. Flavor composition of the flux adds another dimension to the measurements. For the latter case, use is made of inverse beta-decay reactions which are flavor specific to $v_{\mathrm{e}}$ but they must be complemented by flavor-mixed reactions such as elastic scattering from electrons or purely neutral current reactions to test for $v_{\mu, \tau, s}$. At lower energies, the radiochemical experiments (Homestake, GALLEX and SAGE) measure the integrated $v_{\mathrm{e}}$ flux above a threshold $(0.8$ for the former and $0.23 \mathrm{MeV}$, for the latter two). The current, real-time experiments (Kamiokande, SuperKamiokande and SNO) measure energies for a variety of reactions from the neutrinos of the ${ }^{8} \mathrm{~B}(>5 \mathrm{MeV})$ flux using the Cherenkov effect in water and heavy water (SNO). The combination of these experiments is expected to give the first "smoking gun" evidence for or against oscillation of solar neutrinos. The maximum recoiling electron energy from elastic scattering of the principal ${ }^{7} \mathrm{Be}$ line is $665 \mathrm{keV}$; while that from the p-p continuum is only $261 \mathrm{keV}$. The upcoming BOREXINO detector, based on the use of a highly purified liquid scintillator, will give the first direct window on the ${ }^{7} \mathrm{Be}$ flux in real-time using the elastic scattering reaction but is not expected to access the p-p flux because of internal backgrounds at the lowest energies.

Extending the measurements of the p-p neutrinos in the near future is important for several reasons. Even after the completion of all of the above mentioned experiments we still will have a direct measure for $<10 \%$ of the total solar neutrino flux or of its flavor composition. More than $90 \%$ of the solar neutrino flux, namely that from the p-p reaction, will remain to be understood. The region in the Sun where this reaction occurs coincides with that of maximum solar energy production. In standard solar models, the p-p neutrino rate can be precisely $( \pm 1 \%)(1)$ related to solar luminosity. The solar luminosity is well measured; if complemented by experiments designed to measure the total neutrino flux at these low energies an important direct comparison of two measured quantities can be made. Additionally, all of the currently favored, model-dependent solutions (e.g., MSW and vacuum oscillations) make quite different predictions for the flux composition at $\mathrm{p}-\mathrm{p}$ energies. Even if one solution remains strongly favored after the present generation of experiments, a direct measurement of this flux composition can be an important ingredient in establishing the mass-mixing parameters accurately. Except for the large mixing angle MSW solution, these parameters are not likely to be amenable to entirely terrestrially-based experiments.

Detection reactions with accurately known cross sections for the p-p and ${ }^{7} \mathrm{Be}$ neutrinos are available - for the ground state transitions in $\mathrm{Ga}$ and for elastic electron scattering. The continuing gallium-based detectors ( GNO and SAGE), with increased statistics and improved sys- 
tematic errors, will provide more precise measures of the $v_{\mathrm{e}}$ flux for energies $>0.23 \mathrm{MeV}$. Already this flux is known to be at or below the amount allowed by solar models from the p-p reaction alone. A real-time experiment utilizing spectral measurements for the flavor-mixed and precisely known elastic cross-section is needed to provide the essential, but presently missing, sensitivity to the components $v_{\mu, \tau}$ of the low energy flux. An experiment with this capability would also be sensitive to the ${ }^{7} \mathrm{Be}$ neutrinos and, if done by a technique with very different systematics from BOREXINO, could provide an important corroboration of that important measurement.

There are a number of reasons why a real-time, $\mathrm{p}-\mathrm{p}$ experiment is very difficult and no technique has yet been shown to be fully feasible. Principal among the difficulties are the residual radioactivity in the target and the target vessel and the challenge of retrieving energy information for depositions as low as $50-100 \mathrm{keV}$ in a massive target. We have been conducting a series of experimental studies which suggest that a superfluid-based detector may be feasible. We present a synopsis of these studies and their current status.

\section{GENERAL PRINCIPLES OF HERON}

In the HERON detector, the detection reaction is

$$
v_{\mathrm{e}, \mu, \tau}+e^{-} \rightarrow \mathrm{v}_{\mathrm{e}, \mu, \tau}+e^{-}
$$

Fortunately, although its cross-section is low $\left(\sim 10^{-45} \mathrm{~cm}^{2}\right)$ the expected flux is high $\left(\sim 6 \times 10^{10} \mathrm{~cm}^{-2} \cdot \mathrm{s}^{-1}\right.$ for $\mathrm{p}$ - $\mathrm{p}$ neutrinos in the standard solar model) so that $\sim 10$ tons of target would yield about $20 \mathrm{p}$-p events and $7{ }^{7} \mathrm{Be}$ events per day with recoil energies above $50 \mathrm{keV}$. HERON would use superfluid helium as the target material. Helium in the superfluid state is self-cleaning and retains no other atomic species in solution. It has a density of $0.145 \mathrm{~g} \cdot \mathrm{cm}^{-3}$ at $30 \mathrm{mK}$. Consequently it can be made into a very compact target completely free of radioactivity. It has no long lived isotopes and its first excited nuclear state is high, $\sim 20 \mathrm{MeV}$. The technology for handling large volumes of liquid helium is well established and is regularly used in the industrial and research communities. It is inexpensive; at $\$ 30 \mathrm{~K}$ per ton.

Two copious forms of radiation are produced in the liquid by an ionizing particle: UV photons and phonons/rotons (quasi-particles). The photons (15 per $\mathrm{keV})$ are in a narrow range centered at $16 \mathrm{eV}$; an energy at which helium is completely transparent. About $10^{5}$ phonons/rotons per $\mathrm{keV}$ are also produced and propagate ballistically (2) in the superfluid at speeds of $\sim 200 \mathrm{~m} \cdot \mathrm{s}^{-1}$. The signals from both single photons and

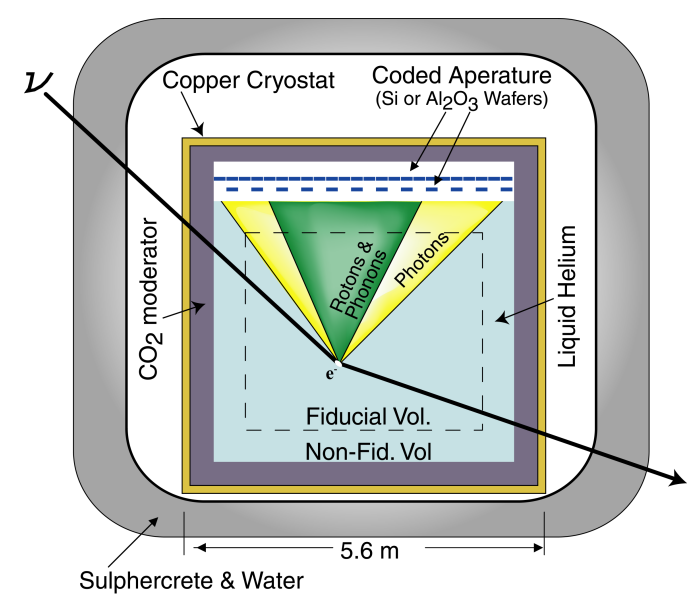

FIGURE 1. A schematic of the HERON configuration.

multiple phonons/rotons will be used to determine the position and energy of each event. These signals are detected on an array of cryogenic wafer-calorimeters placed above the surface of the liquid. The prompt photons are absorbed directly by the wafers providing a fast trigger and can be used to find the event position. The wafers of silicon or sapphire are arranged in two patterned planes to form a coded aperture array. The distribution of photons hitting the array is used to determine the location of the event in a manner similar to that used in $\mathrm{x}$-ray astronomy and tomography. The delayed phonons/rotons are detected through the secondary process of quantum evaporation (3) and adsorption (4); this signal is combined with that of the photons for energy determination and the relative timing among the wafers is used as a check on event position.

While the target itself can be made completely free of background producing materials, backgrounds emanating from the containment cryostat must be tagged and subtracted. This is to be done by logically dividing the helium volume into a fiducial and non-fiducial region and making a statistical subtraction. Figure 1 represents a generalized view of a detector based upon these principles.

\section{EXPERIMENTS ON SINGLE PARTICLE DETECTION}

We have carried out a series of experiments using stopping electrons and alpha particles in order to acquire a quantitative understanding of the energy loss process in superfluid. The experiments have been carried out in a test cell which contains $\sim 3$ liters of superfluid typically held at $30 \mathrm{mK}$ by a dilution refrigerator. The interior of 
the cell is instrumented with a variety of devices which can be re-configured appropriately for each experiment. Besides thermometers, heat pulsers and liquid level sensors, there are small, superconducting stepper motors for positioning and moving radioactive sources within the liquid volume. The sources were low activity ${ }^{241} \mathrm{Am}$ for 3-5 MeV alphas and ${ }^{113} \mathrm{Sn}$ for $364 \mathrm{keV}$ electrons. X-rays from ${ }^{55} \mathrm{Fe}$ as well as from the $\mathrm{Sn}$ and Am sources were used for calibration.

Thin silicon or sapphire wafers ranging in size from 5 $\mathrm{cm}$ dia. discs to $1 \times 2 \mathrm{~cm}$ rectangles, were mounted above the liquid surface to be used as calorimeters. The temperature rise in these wafers was measured by Ir-Au thinfilm superconducting transition edge sensors (TES)(5) deposited on the surface of the wafers. The TES devices were run in a self-biasing mode and read out with conventional SQUID electronics. The wafers were kept free of superfluid film by a "film burning" device (6). The signals from the phonons/rotons and photons are detected as follows.

The ballistically propagating phonons/rotons which reach the free surface of the liquid and whose energy is greater than the helium-helium binding energy can cause quantum evaporation. This is a process in which one phonon or roton can evaporate one helium atom and does so about $30 \%$ of the time (3). Energy and momentum conservation at the liquid surface place constraints on the incident roton angles which can cause evaporation (7). The helium binding energy is $0.7 \mathrm{meV}$ and the typical phonon/roton energies involved are $\geq 0.8 \mathrm{meV}$. Thus in the case of a charged particle in the $50 \mathrm{keV}$ range (ie a recoil electron from a neutrino event) a very large number of atoms are involved, and are subsequently evaporated. The evaporated atoms are adsorbed onto the wafer and their deposited binding energy produces a heat pulse in the TES. The binding energy of the helium to the substrate is approximately a factor of ten higher than that of the helium-helium binding energy, thus there is an effective amplification in the evaporation-adsorption process.

The scintillation (UV photons) result from the radiative decay of helium dimers formed along the track and have energies in the range of $14-20 \mathrm{eV}$, but peaked at $16 \mathrm{eV}$. Since the first excited state of atomic helium is at $20 \mathrm{eV}$ the liquid is transparent to lower energies. The absorption of photons in the silicon and sapphire wafers create a prompt heat pulse in them. The two contributions, photons and adsorbed helium atoms, can be distinguished by their different times of arrival on the wafer. In the results described here, the energy threshold of the wafers was $300 \mathrm{eV}$, so single photons were not detectable. The principal results for alpha particles, including evidence for particle directionality, are discussed elsewhere (8).

The ${ }^{113} \mathrm{Sn}$ had an activity of a few nano-curie so that single electrons could be distinguished. A $364 \mathrm{keV}$ elec-

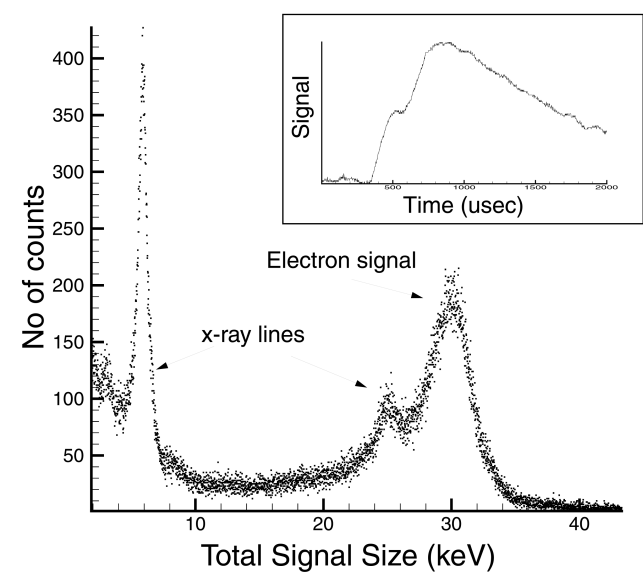

FIGURE 2. Inset: a typical $364 \mathrm{keV}$ electron pulse from superfluid helium; the initial rise is due to photons and the later one to evaporation by phonons/rotons. The main figure is the energy spectrum for $364 \mathrm{keV}$ electrons in superfluid helium; also shown are the 6 and $25 \mathrm{keV}$ x-ray calibrations.

tron is produced by isomeric transition in ${ }^{113} \mathrm{In}$ following electron capture; a $25 \mathrm{keV} \mathrm{x}$-ray is also produced and is used as one of the calibration points for the wafers. For the data illustrated in Figure 2, the source was $\sim 5 \mathrm{~cm}$ below the wafer and the time delay difference can be clearly seen in the pulse shape even for that very short distance. Also shown in the figure is the measured single electron spectrum.

We find that the division of the electron's initial energy is $25 \%$ into UV photons (or 15 photons $/ \mathrm{keV}$ ) and $10 \%$ into detectable phonons/rotons (or $10^{5}$ phonons $/ \mathrm{keV}$ ). This contrasts with the $8 \%$ and $40 \%$, respectively, for alpha particles. It should be noted that the energy fraction represents the energy deposited directly into the helium into $4 \pi \mathrm{sr}$ and has been corrected for geometrical factors. However in the case of the phonon/roton component, this energy also differs from what is measured on the bolometers due to the effective gain from binding energy differences. These results agree well with a model in which the formation of excited helium dimers in the close vicinity of the track, and their subsequent de-excitation by radiation or collision, plays a central role in determining the above energy fraction. (9).

\section{WAFER SENSITIVITY}

For the coded aperture array to be useful for recoil energies as low as $50 \mathrm{keV}$ in a large detector, the wafers must be able to detect with high probability a single 16 $\mathrm{eV}$ photon. In our prototype cell so far using transition edge sensors on our $5 \mathrm{~cm}$ dia.. wafers our threshold was 
$\sim 20$ photons. Thus to ensure that our modified detection technique is successful we must develop somewhat larger wafer/calorimeters with a threshold sensitivity of at least $10 \mathrm{eV}$.

We have taken as a benchmark event for establishing the performance requirements of a large-scale neutrino detector, the detection and measurement of an approximately $50 \mathrm{keV}$ electron, 4 meters below the surface of a container of superfluid helium. Wafers of about 150 $\mathrm{cm}^{2}$ with a $10 \mathrm{eV}$ threshold are needed to meet the performance requirements of a full-scale neutrino detector; however, the successful development of a neutrino detector does not depend upon any anticipated improvements in the performance of transition edge sensors. Rather, we have shifted to a very promising and rapidly developing different technology which should allow wafers with the necessary increase in size and improvement in sensitivity. The technology is that of the magnetic micro-calorimeter which would replace the TES as the sensor on the wafers. A magnet sensor for calorimetry consists of a material with a strong temperature-dependent magnetization and a means to measure that magnetization. Small amounts of energy deposited in the calorimeter cause a magnetic flux change which is measured with dc SQUID electronics. For low temperature operation we have used a system of dilute paramagnetic rare earth ions in metallic gold. The initial development experiments have been carried out in Heidelberg and at Brown using Er as the paramagnetic ions.

To see the power of the technique it is useful to cite an early result of our collaborating colleagues in Heidelberg in which they had achieved at $25 \mathrm{mK}$ a resolution of $135 \mathrm{eV}$ at $6 \mathrm{keV}$ and excellent linearity (11). The resolution of $135 \mathrm{eV}$ is, by itself not exceptional, but the fact that the calorimeter in the measurement had a heat capacity of about $4 \times 10^{-9} \mathrm{~J} / \mathrm{K}$ makes the result important. This heat capacity is comparable to that of $15 \mathrm{~cm}^{3}$ (the volume of a $20 \mathrm{~cm}$ diameter, $0.5 \mathrm{~mm}$ thick wafer) of silicon at $50 \mathrm{mK}$. There are a large number of experimental parameters that have not been optimized for our particular application: physical size, spin concentration, magnetic field, temperature, bandwidth and filtering, flux coupling to the SQUID, etc. We base our confidence in being able to predict the performance achievable with a magnetic calorimeter on the fact that it is a well defined and well characterized thermodynamic system. The response of the system to an energy input can be calculated. Measurements so far are in good agreement with theory. Our expectation is that we shall eventually come close to the thermodynamic noise limit set by the temperature, heat capacity, coupling to the thermal reservoir and bandwidth of measurement.

Significant progress continues and just recently a major step forward was made. A resolution of $13 \mathrm{eV}$ at
$6 \mathrm{keV}$ has now been achieved by the Brown-Heidelberg collaboration with a magnetic calorimeter having a heat capacity of about $1 \times 10^{-12} \mathrm{~J} / \mathrm{K}(10)$. Since the sensitivity varies roughly as $\mathrm{C}^{-1 / 3}$ for magnetic calorimeters, we require a factor of 5 improvement over present performance. Such an improvement is well within that predicted to be achievable with better calorimeter design. Based on the experience gained so far, we believe that calorimeter performance adequate for large-area wafers with a $10 \mathrm{eV}$ threshold is feasible.

\section{EVENT LOCATION AND BACKGROUNDS}

In liquid helium the range of $\mathrm{p}-\mathrm{p}$ and ${ }^{7} \mathrm{Be}$ recoil electrons is less than $2 \mathrm{~cm}$. On the scale of the detector (volume $\sim 60 \mathrm{~m}^{3}$ ), these events are effectively point sources of photons and phonons/rotons. In contrast, most of the background gamma-rays, which originate in the cryostat, make spatially extended energy depositions by multiple Compton scattering. Although there is always a small number of single Compton scatterings, the major contribution to background is from multiple depositions originating from an initial gamma-ray. To suppress the background, multiple Compton scattering events must be identified and the spatial distribution of point energy depositions obtained. The background from point depositions can be determined statistically using the measured energy and spatial distribution of such events in the nonfiducial region. A knowledge of the position of the event in a large detector is essential not only for background rejection and diagnostics but also for the determination of the energy of the event.

Coded aperture arrays, mentioned above, are often used to determine the position of a source in circumstances where the radiation is difficult or impossible to focus or is of low intensity, such as for x-rays. In simple terms, the original (12) principle of operation is based on the concept of decoding an image formed on an image plane by the "shadow" of a mask constituting a multiple hole pin-hole camera where the mask pattern and plane separations are known. A large modern literature exists, and a large variety of mask patterns are employed (13).

In our application there are important differences and simplifications in implementation. There are, of course, image and mask planes but here the "pixels" are wafercalorimeters, with the lower mask plane having roughly $50 \%$ or more transmission. Unlike the usual method of coded apertures, the wafers forming the mask for HERON will also be active calorimeters and be used in both position finding and energy determination. Thus, no signal is lost by the presence of the mask. Depending on 
photon statistics, the distribution of their signals on the image plane uniquely determines the source position. The accuracy with which the array is able to measure event location can be directly tested in-situ for a large detector by using a movable radioactive source.

To evaluate potential performance of this application, we have initiated Monte Carlo simulations in which the distribution of photons incident on the wafers from a source anywhere in the detector is used to determine the most probable position of an event based on a maximum likelihood method. In the calculations we have assumed the wafers have an energy threshold sufficiently low to detect single $16 \mathrm{eV}$ photons. We have simulated a HERON-sized array using a model in which the image plane consists of $1600,150 \mathrm{~cm}^{2}$, densely packed wafers, separated by $50 \mathrm{~cm}$ from the mask plane of 800 wafers. These early simulations suggest that it may be possible to determine the position of an energy deposition by an electron over most of a $60 \mathrm{~m}^{3}$ cube of liquid with a resolution of $10-15 \mathrm{~cm}$ FWHM provided more than about 75 photons hit the array. The 75 corresponds to an energy of 30 $\mathrm{keV}$ for an event near the top and $75 \mathrm{keV}$ near the bottom of the fiducial volume. We have not yet optimized the parameters of the array (among them: pixel size, mask pattern, plane spacing, etc.) nor the search algorithms. Additionally, a thorough study of the background involving multiple Compton scattering events, which should be distinguished by the poor quality of the determination of event location, must be made.

Devising a strategy for the control of backgrounds is also a key issue. For this purpose we have carried out Monte Carlo simulations based on assumptions of detector performance and knowledge of expected sources. Because the signal signature is a single recoil electron appearing in the detector volume, Compton scatterings of gamma-rays are the most dangerous. (The relative pulse heights of photon vs. phonon initiated signals is a discriminant against heavily ionizing deposition.) At the energies from likely sources, Compton scatters constitute more than $90 \%$ of $\gamma$-ray conversions in liquid helium. The major sources are from cosmogenically produced isotopes in the cryostat materials while on the surface of the Earth and from heavy elements ( $U$ and $T h$ ) in the cryostat or any material placed in immediate contact with the helium. Decays from such activities located in the wafer planes or from slow neutron captures elsewhere are minor sources. Several steps can be taken to control the signal to background. Those we have included in our simulations are: minimization of cosmogenics by control of exposure and storage time (two months above and two years below ground), minimizing $\mathrm{U}$ and $\mathrm{Th}\left(<10^{-12} \mathrm{~g} / \mathrm{g}\right)$ in the copper of the cryostat by electroforming, providing a cryostat liner ("moderator") of low U/Th content $\left(<10^{-14} \mathrm{~g} / \mathrm{g}\right)$ and providing a non-fiducial volume of he- lium sufficient to allow a statistical background subtraction. Liquid helium is not an efficient absorber of high energy ( $\sim 2 \mathrm{MeV}$ ) $\gamma$-rays; however, while it is possible to use an excess volume of liquid helium itself as an attenuator of these external $\gamma$ 's to do so makes the size impractically large. Conversely, the detector dimensions are large enough that lower energy ( $\leq 1 \mathrm{MeV}) \gamma$ 's produce a multiplicity of spatially well separated Compton recoils. The main purpose of the "moderator" then is energy degradation of the higher energy $\gamma$ 's to produce a more distinctive background signature as well as some attenuation.

In a typical simulation to study background, a 10 ton cylindrical copper cryostat ( $5.6 \mathrm{~m}$ dia.x $5.6 \mathrm{~m}$ ht.) with a 25 $\mathrm{cm}$ thick moderator of $\mathrm{CO}_{2}$ (or frozen non-polar liquid) and containing a total ( fiducial plus non-fiducial volume ) of $\sim 18$ tons of superfluid helium was used. The cosmogenic spectrum and rates in the material were taken from double-beta decay and dark matter experiments (14) as per the above mentioned storage conditions $(45 \mu \mathrm{Bq} / \mathrm{kg})$. The levels of $\mathrm{U}$ and $\mathrm{Th}$ in the materials was chosen to make their overall contribution to the background 10$20 \%$ of that from the cosmogenics. Single photon sensitivity and the ability of the coded aperture array to perform as described was assumed. High statistics runs were made to be the equivalent of six month's data taking. A variety of different relative sizes and shapes of fiducial and non-fiducial volumes were used. Typical cuts on events required energy depositions $\leq 800 \mathrm{keV}$ and single or multiple Comptons within the assumed spatial resolution from the coded aperture array. The resulting deposited energy spectra in the fiducial and non-fiducial regions are remarkably similar down to $<100 \mathrm{keV}$ thus facilitating a direct bin-by-bin subtraction. The normalization is taken from the Monte Carlo. Since the division between fiducial and non-fiducial volumes is done in software, in a real detector that division could be varied to check for consistency of subtraction. As examples from the simulation with a fixed total volume, a fiducial to non-fiducial volume ratio of 1:1 gave an unsubtracted signal to background of 3.5:1 while a volume ratio of 1.5:1 gave signal to background of $0.8: 1$. We believe we have taken realistic assumptions as Monte Carlo input so if the conditions of performance and activity assumed can be achieved then this background strategy should be adequate for obtaining the electron recoil rate and spectrum from low energy solar neutrinos.

\section{SUMMARY AND CONCLUSIONS}

At each stage in the history of solar neutrino research imaginative and unique new experimental tools have been successfully developed to achieve the task then at hand. 
There is now increasing interest to study the lowest energy part of the neutrino spectrum which constitutes $>90 \%$ of the total flux. However, real-time detection of $\mathrm{p}$-p and ${ }^{7} \mathrm{Be}$ neutrino interactions presents severe experimental challenges to developing a detector because of the backgrounds inherent in the target material and the material surrounding the target. Another concern is the very low energy signal that must be extracted from a massive target. Once again, a new technique is needed.

We have presented results on the progress made so far toward developing a new type of detector, HERON, to have this capability. Its essential feature is the use of superfluid helium as the target material. Only at these very low temperatures can helium be made entirely free of internal backgrounds. The goal of our experiments and studies so far has been to gain sufficient knowledge of the conditions needed to establish feasibility of the new technique. Several of these conditions have either been met or appear to be achievable. We now have a thorough understanding of the processes by which low energy electrons can be detected using both photons and quasiparticles (phonons/rotons). We have developed calorimeter sensors able to detect both phonon and quasi-particle induced signals. The sensitivity of these devices is showing rapid improvement. We have carried out detailed studies of strategies for controlling externally produced backgrounds. The initial simulations of position finding within a detector by the new coded aperture method shows considerable promise. Recently, we have upgraded our simulation capability for optimizing the array and testing with what precision an event can be found with no prior information as to its approximate location in the entire detector and with what signal to background selectivity. For neutrino experiments at these low energies, we believe that the ability to locate an event position inside a detector is an essential control on backgrounds from sources outside the target material itself. The requirements for radioactivity in the cryostat and moderator are stringent but appear possible. Presently, we are concentrating on two topics which are fundamental to the success of the superfluid technique: achieving single, $16 \mathrm{eV}$ photon sensitivity on wafer sensors and demonstrating the efficacy of the coded aperture for event location and background signature recognition.

\section{REFERENCES}

1. Bahcall, J.N. and Krastev, P.I., Phys. Rev. D53, 4211 (1996).

2. Hendry, P.C. and McClintock, P.V.E., Cryogenics 27, 131 (1987) Lowering the natural concentration of ${ }^{3} \mathrm{He}$ reduces roton scattering. Using a single pass with the Hendry-
McClintock heat flush technique during detector filling we have reduced the ${ }^{3} \mathrm{He}$ content of the superfluid to $1: 10^{-9}$.

3. Brown, M and Wyatt, A.F.G.,J. Phys.: Condens Matt., 2, 5025 (1989).

4. Adams, J.S. et al, Phys. Lett. B341, 431 (1995).

5. Bühler, M. et al, Proceedings of 7th International Workshop on Low Temperature Detectors, 149 (1997), Editor: S. Cooper (Max Planck Institut, Munich). We wish to thank the von Feilitzsch Group for the deposition of these thin films on our wafers.

6. Torii, R. et al, Rev. Sci. Instr. 63, 230 (1992).

7. Bandler, S.R. et al, Phys. Rev. Lett. 68, 2429 (1992).

8. Bandler, S.R. et al, Phys. Rev. Lett. 74, 3169 (1997); Bandler, S.R. et al, Nucl. Instr. \& Meth. A370, 578 (1996).

9. Adams, J.S., Kim, Y.H., Lanou, R.E., Maris, H.J. and Seidel, G.M., J. Low Temp Phys., 113, 1121 (1998). See also Adams, J.S. et al, (To appear in Nucl. Phys.B: Proceedings of Workshop on Low Temperature Detectors ("LTD8") Dalfsen, Holland; in press (1999)).

10. Schönefeld, J. et al, (To appear in Nucl. Phys. B: Proceedings of Workshop on Low Temperature Detectors ("LTD8") Dalfsen, Holland; in press (1999)).

11. Fleischmann, A., et al, (To appear in Nucl. Phys.B: Proceedings of Workshop on Low Temperature Detectors ("LTD8") Dalfsen, Holland; in press (1999)).

12. See for example, Dicke, R.H., Astroph. J. 153, L101 (1968).

13. See for example, Fenimore, K.E. and Cannon, T.M., Applied Optics 17, 337 (1978).

14. Private communication: Zerle, L. (CRESST collaboration); Heuser, G. Low-Level Measurements of Radioactivity in the Environment (Proc. of 3rd Intl. Sch., 69 (1994), Editor: M. Garcia-Leon (World Sci. Publ.).

Supported in part by DoE DE-FG02-88ER40452 \& NSF PHY-9870276 